\title{
The role of p53 in limiting somatic cell reprogramming
}

\author{
Yan Liu ${ }^{1}$, Ruben Hoya-Arias ${ }^{1}$, Stephen D Nimer ${ }^{1}$ \\ ${ }^{I}$ Molecular Pharmacology and Chemistry Program, Sloan-Kettering Institute, Memorial Sloan-Kettering Cancer Center, New \\ York, New York 10065, USA \\ Cell Research (2009) 19:1227-1228. doi:10.1038/cr.2009.121; published online 2 November 2009
}

The first successful generation of induced pluripotent stem (iPS) cells from somatic cells was accomplished by introducing four genes into the cell, Oct3/4, Sox2, Klf4, and c-myc [1]. While a tour-de-force, this approach to iPS cell generation is inefficient, and unlikely to be directly translated into therapeutic use since it involves the use of retroviruses to introduce these genes into the cell. Subsequent studies have used non-integrating genetic elements, chemical compounds, or proteins rather than DNA to bypass concerns about retroviral insertional mutagenesis [2-5].

Five papers published in Nature last month provide much additional information about improving the efficiency of iPS cell derivation from somatic cells; however, they also sound notes of caution regarding the clinical utility of their approach. Zhao et al. first linked inhibition of the tumor suppressor protein $\mathrm{p} 53$ with the efficiency of generation of iPS cells [6], and these new studies shed light on how the p53 pathway hinders reprogramming, and demonstrate that eliminating p53 function greatly improves iPS cell generation from both mouse and human cells.

Somatic cell reprogramming is a stress process, thus, it is logical that p53 could play a pivotal limiting role. During this process, somatic cells

Correspondence: Stephen D Nimer

Tel: +1-646-888-3040; Fax: +1-646-422-0246

E-mail: s-nimer@mskcc.org activate the $\mathrm{p} 53$ pathway and induce apoptosis, senescence, or cell-cycle arrest, all which appear to contribute to the low efficiencies seen. In the report by Marion et al., the act of reprogramming was shown to trigger DNA damage, which could be tolerated by the cell if p53 activity was abrogated during the time of iPS cell generation. Otherwise, the reprogramming process activates the DNA damage response and induces p53-dependent apoptosis [7]. Senescence represents another barrier to reprogramming as it is easier to generate iPS cells from early passage fibroblasts than from late passage or partially senescent ones. Aging represents yet another barrier, and p53 has been implicated in this process as well. Upregulation of the INK4/ARF locus occurs during the aging process, which may explain why reprogramming is also less efficient if cells isolated from older animals are used. Li et al. demonstrated that activation of the INK4/ARF locus is another barrier for iPS cell reprogramming. The INK4/ARF locus is silenced in iPS cells and in embryonic stem cells [8], and this group has established that inhibiting the INK4/ARF locus has a profound positive effect on the efficiency of iPS cell generation.

The reports from Yamanaka's and Belmonte's groups provide more information on $\mathrm{p} 53$ 's function during iPS cell generation. Hong and colleagues introduced Sox2, Klf4, and Oct4 (without $c-m y c$ ) into p53 null fibroblasts, while Kawamura and colleagues introduced
Sox2 and Oct4; both observed an enhanced efficiency in generating iPS cells $[9,10]$. Hong et al. were also able to use p53 null (but not wild type) terminally differentiated $\mathrm{T}$ cells to generate iPS cells [9]. By introducing wild type p53 and a series of $\mathrm{p} 53$ mutant cDNAs into the p53 null murine cells, they showed that while wild type 553 inhibits iPS cell generation, transactivation-defective forms of p53 had no effect. Suppression of the p53 pathway also increased the efficiency of human iPS cell generation. So they performed gene expression profiling to identify which genes downstream of $\mathrm{p} 53$ were limiting iPS cell generation. 34 genes were identified that were regulated by $\mathrm{p} 53$ in both mouse and human fibroblasts. Of these, $M D M 2$ and $p 21$ clearly affected stem cell reprogramming, with $\mathrm{p} 21$ mimicking the effect of p53 and MDM2 mimicking p53 suppression. The report from Belmonte's group similarly showed the importance of $\mathrm{p} 21$ in this process. Thus, the p53-p21 pathway limits the generation of iPS cells, while two other p53 regulated genes, BTG2 and matrin 3, apparently do not.

We, and others, have shown that p53 negatively regulates HSC self-renewal [11]. Furthermore, although multipotent hematopoietic progenitor cells lack the ability to self-renew, in cells lacking the INK4/ARF locus, the absence of p53 imparts a self-renewal signal to these progenitors [12]; this seems somewhat analogous to its effects on reprogramming. Thus, loss of INK4A/ARF and 
p53 may serve to facilitate somatic cell reprogramming by enhancing the selfrenewal capability of the cell.

It appears as though iPS cell generation closely resembles the process of tumorigenesis. Cancer stem cells might actually arise through a reprogramminglike process, gaining the ability to self-renew and becoming more highly transformed with the loss of $\mathrm{p} 53$ function. It is important to note that the ES cells generated by targeting of p53 form teratomas when transplanted into immunodeficient mice [9], and clearly the permanent suppression of $\mathrm{p} 53$ may significantly impair the quality of the iPS cells generated due to enhanced genomic instability. Transient lowering of p53 levels may be more useful in generating integration-free iPS cells, and in the report by Utikal et al., the acute loss of p53 was shown to allow iPS cell to be generated from cells that had failed to reprogram [13]. They observed that primary mouse fibroblasts with low-level p19ARF expression or those lacking p53 expression yield iPS cells with up to 3 fold faster kinetics and a much higher efficiency than wild type cells. In fact, immortal fibroblasts that lack an intact arf-p53 pathway can be reprogrammed with almost unit efficiency. While the $\mathrm{p} 53 /$ arf and $\mathrm{E} 2 \mathrm{~F} / \mathrm{Rb}$ pathways have both been implicated as tumor suppressors in cancer prevention, knock down of $\mathrm{Rb}$ did not enhance iPS cell generation, while knock down of p53 clearly did.

Oncogene expression can trigger a variety of cellular self-defense mecha- nisms including apoptosis, senescence, and cell cycle arrest. We recently identified another role for $\mathrm{p} 53$, namely its ability to regulate the quiescence of hematopoietic stem cells [11]. There is increasing evidence that "tumor suppressor proteins" may play a key role in regulating the cell cycle entry of this population of cells. The role that quiescence plays in limiting iPS cell generation has not been broadly examined but it may be another attribute regulated by the p53/arf pathway, which can affect iPS cell generation. Because reprogramming and oncogenic transformation share many similarities, knocking down critical barriers to reprogramming may promote the process of transformation. Even though our efforts to reprogram somatic cells are in their infancy and our knowledge about transformation is only several decades further along, the knowledge gained about one process can only contribute to our greater understanding of the other.

\section{References}

1 Takahashi K, Yamanaka S. Induction of pluripotent stem cells from mouse embryonic and adult fibroblast cultures by defined factors. Cell 2006; 126:663676.

2 Stadtfeld M, Nagaya M, Utikal J, Weir $\mathrm{G}$, Hochedlinger K. Induced pluripotent stem cells generated without viral integration. Science 2008; 322:945949.

3 Okita K, Nakagawa M, Hyenjong H, Ichisaka T, Yamanaka S. Generation of mouse induced pluripotent stem cells without viral vectors. Science 2008;
322:949-953.

4 Huangfu D, Osafune K, Maehr R, et al. Induction of pluripotent stem cells from primary human fibroblasts with only Oct4 and Sox2. Nat Biotechnol 2008; 26:1269-1275.

5 Zhou H, Wu S, Joo JY, et al. Generation of induced pluripotent stem cells using recombinant proteins. Cell Stem Cell 2009; 4:381-384.

6 Zhao Y, Yin X, Qin H, et al. Two supporting factors greatly improve the efficiency of human iPSC generation. Cell Stem Cell 2008; 3:475-479.

7 Marion RM, Strati K, Li H, et al. A p53-mediated DNA damage response limits reprogramming to ensure iPS cell genomic integrity. Nature 2009; 460:1149-1153.

8 Li H, Collado M, Villasante A, et al. The Ink4/Arf locus is a barrier for iPS cell reprogramming. Nature 2009; 460:1136-1139.

9 Hong H, Takahashi K, Ichisaka T, et al. Suppression of induced pluripotent stem cell generation by the p53-p21 pathway. Nature 2009; 460:1132-1135.

10 Kawamura T, Suzuki J, Wang YV, et al. Linking the p53 tumour suppressor pathway to somatic cell reprogramming. Nature 2009; 460:1140-1144.

11 Liu Y, Elf SE, Miyata Y, et al. p53 regulates hematopoietic stem cell quiescence. Cell Stem Cell 2009; 4:3748.

12 Akala OO, Park IK, Qian D, et al. Longterm haematopoietic reconstitution by Trp $53^{--}$p $16^{\text {Ink4a-- }}$ p19 $19^{\text {Arf- }-}$ multipotent progenitors. Nature 2008; 453:228232.

13 Utikal J, Polo JM, Stadtfeld M, et al. Immortalization eliminates a roadblock during cellular reprogramming into iPS cells. Nature 2009; 460:1145-1148. 